\title{
Application of the Cellular Automata Method to Simulation of Multiple Pit Similar Defects
}

\author{
BOHDAN RUSYN ${ }^{1, a^{*}}$, ROXANA TORS ${ }^{\prime} K A^{2, b}$ and \\ ROSTYSLAV KOSAREVYCH ${ }^{3, C}$ \\ ${ }^{1}$ Karpenko Physico-Mechanical Institute of NASU 5, Naukova Str., Lviv, Ukraine \\ Technical University of Radom, 29, Malczewskiego Str., Radom, Poland \\ ${ }^{2}$ Karpenko Physico-Mechanical Institute of NASU 5, Naukova Str., Lviv, Ukraine, \\ ${ }^{3}$ Karpenko Physico-Mechanical Institute of NASU 5, Naukova Str., Lviv, Ukraine \\ arusyn@ipm.Iviv.ua, ${ }^{b}$ roxana.torska@gmail.com, ${ }^{\mathrm{c}}$ kosarevych@ipm.Iviv.ua
}

Keywords: cellular automata; numerical simulation; pitting; corrosion; modeling

Abstract. Simulation results from using the cellular automata as an example of defects on the surface of
austenitic stainless steel 08Cr18Ni10Ti are presented in this paper. Practically confirmed the feasibility
of the proposed method for simulation modeling of corrosive process of steel in neutral environments.

\section{Introduction}

Cellular automaton is a discrete dynamic system represented by a set of equivalent cells in the same way interconnected. All cells form the so-called lattice of cellular automata. Lattices can be of different types have different dimensions and shape of cells.

Each of these cells is a finite automaton, its states are determined by the states of neighboring cells and its own state in a given time. The evolution of each cell corresponds to a change of its state at each iteration step and is in sync for the whole lattice, according to predefined transition rules $[1,2]$.

In the work of Wolfram [3] is shown that cellular automata as discrete dynamical systems share many common properties of continuous dynamic systems, but the cellular automata cells provide a simple structure. Due to this feature, they are a good mathematical tools for creating simulation models of the dynamics of pitting damage on the metal surface.

The basic assumption of our proposed model based on cellular automata is that corrosion is limited by diffusion between mobile agents and unprotected metal. Passive layer acts as a perfect barrier. It is assumed that growth in the act of pitting simulation already initiated.

In this approach, the model is represented as a finite two-dimensional cellular automata on a rectangular lattice, where the system metal/film/aggressive environment describes a set of unit cells and a set of specified conditions, which may be in these cells. For basic physical and chemical processes, such as mass-transfer, dissolution of metal, passivation and repassivation, sets the rules under which the cellular automata cells interact.

At each step of the modeling procedure is determined by the configuration of cells obtained and calculated the amount of dissolved metal as a function of time. It is important that the simulation here is arbitrary.

Local transition rules [4] consider anodic reaction, diffusion, passivation, repassivation: 1) anode reaction modeling the behavior of cells when dissolved metal inside defect; 2) passivation processes, reflect the transition of cells that are responsible for the metal, the state of passivity to activity state when the acidity of the medium increases; 4) a description of diffusion processes modeling movement of the metal cells in the aggressive environment. 


\section{Origin of the Pitting Corrosion}

Pitting corrosion - corrosion type, which are subjected to extremely passive metals and alloys. This type of corrosion destruction observed in nickel, zirconium, chromium-nickel, chromium, aluminum and others alloys. At pitting corrosion only some areas of the surface are destructed, which are formed deep affection - pittings (point defects). Pittings are formed suddenly as a result of random changes in the concentration of the liquid or the presence of small surface scratches or defects. Some defects can become inactive due to walk convective flow, while others can grow to very large sizes, with the formation of stagnant zones that and continue to grow over time. Defects are usually grow in the direction of gravity, because for rapid pit growth requires a high concentrated solution. Most defects is growing down from a horizontal surface, corrosive ultimately through the material. Less defects formed on vertical plane and very little - at the top.

Evolution of pit similar defect is divided into three stages: nucleation, growth and repassivation.

Pitting nucleation stage (formation micro-area of active dissolution) begins at achieving pitting potential $E_{p i t}$ in the oxide film defects, or heterogeneous inclusions in the metal.

Metastable phase of growth - the process of ionization of the metal is a high speed and is accompanied by hydrolysis, which reduces the level of $p H$ in pit defect and increases the rate of dissolution of the oxide. The concentration of the electrolyte solution and its composition in depth of pitting different from the bulk (increased concentration of metal ions and anions, including activators). Thus, the conditions for recovery of hydrogen ions, i.e. corrosion with hydrogen depolarization, on the one hand, and the inhibition of diffusion of pitting rises - on the other.

Sometimes the next stage is pitting repassivation (termination of the rate), resulting shift a pit potential in a passivity area. Thus, pitting corrosion occurs when the conditions of transition metal in the passive state (e.g., oxidizing solution is - chromates and nitrates, etc.), while in the presence of corrosive environment activator ions in a concentration equal to or greater than the critical. The critical concentration of halide ions depends on the nature of the metal, the presence of other anions in solution and so on.

Many factors influence on the transition from one pitting growth stage to another, among them are such as termination of diffusion processes associated with the formation of corrosion products, the degree of destruction of the protective film, the pitting radius and passivity salt film [5, 6]. For a complete description of the processes of pit similar defect, physical model should cover all the processes involved in its formation.

\section{Results and Discussion}

Studied the corrosion behavior of stainless steel $08 \mathrm{Cr} 18 \mathrm{Ni} 10 \mathrm{Ti}$, in the $3 \% \mathrm{NaCl}$ at room temperature. All polarization tests were performed at a speed scanning of $0.5 \mathrm{mV} / \mathrm{s}$.

The study was conducted as follows. Sample surfaces were polished in the air, subsequently, the sample polarized at $+810 \mu \mathrm{V}$. On passivated sample fixed of cathodic polarization dependence. After that exclude the polarization kept the sample to stabilization and fixed the anodic branch. In this method of surface retrieval is the disadvantage that the sample may hydrogenized. Potential of 08Cr18Ni10Ti at all temperatures eventually ennobled, so this steel is prone to passivation, which significantly complicates the task of modeling of defects on the surface.

A comparative analysis of images about hundred simulated pittings with real pit defects obtained on stainless steel during its digestion. Real defects in shape can be divided into two groups: open and partially closed defects corresponding to stable and metastable pittings. The nature of their formation is explained by different thickness and resistance of the oxide film on the polished and brushed surfaces, and - acidity aggressive environment.

Since profilometric methods (as contact and contactless) and microscopy have certain geometric constraints to the form of the objects, the study of the size and shape partially closed pittings is problematic and in this paper is not considered. 
Investigated kinetics of defect growth consistent with the experimental results. In addition, the simulated cases for different number of defects formed at the same time, reflecting the characteristic pattern of the actual physical process by the example of pitting corrosion during the simulation procedure.

In order to investigate the behavior of cellular automata, conducted a series of four acts simulations, each of them consisted of 50 acts simulation. According to the results, the nature of defects during simulation for various different default number of potential defects - were different forms of modeled pit similar defects and their location on the surface after completion of the simulation. In Fig. 1, respectively, are the real defects image $(1, a)$ and simulated $(1, b)$ defects using the proposed models based on cellular automata. The value of $p H$ for all cases was the same and was 7 .

Comparison of the results was based on data obtained not only by visualization, but also when comparing the actual cathode-anode polarization curve constructed for stainless steel $08 \mathrm{Cr} 18 \mathrm{Ni} 10 \mathrm{Ti}$ among $3 \% \mathrm{NaCl}$ at room temperature (Fig. 2.) And cathode-anode polarization curve obtain during the modeling process.
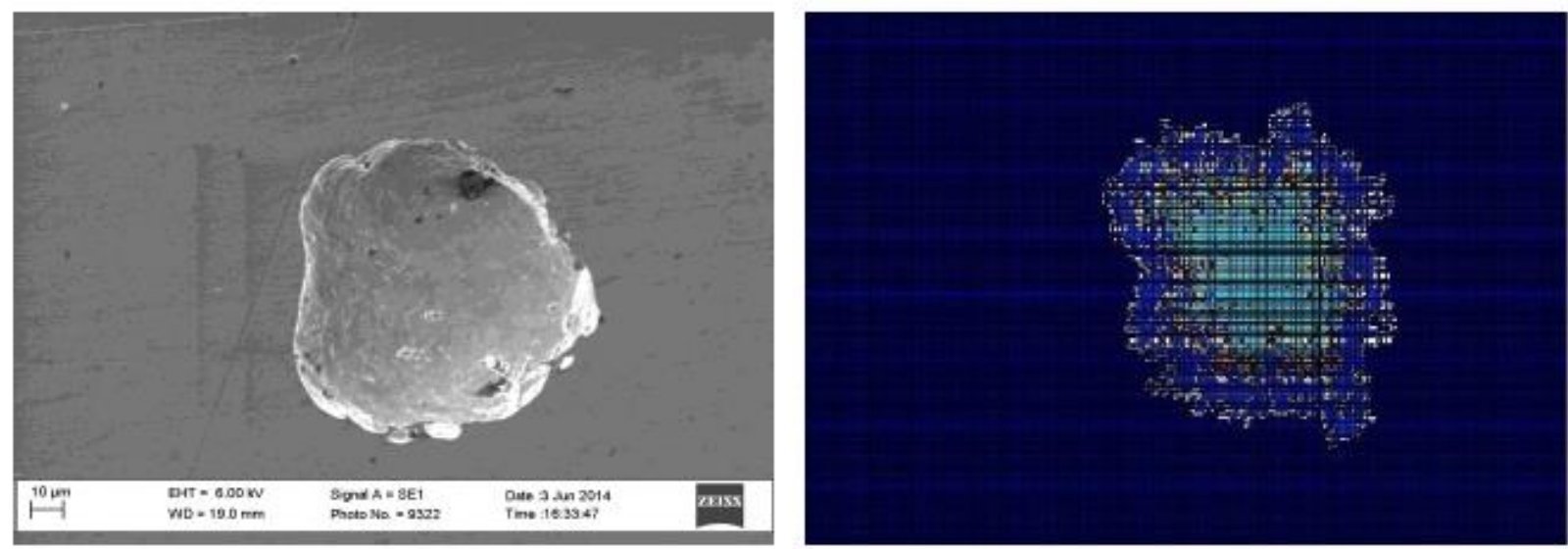

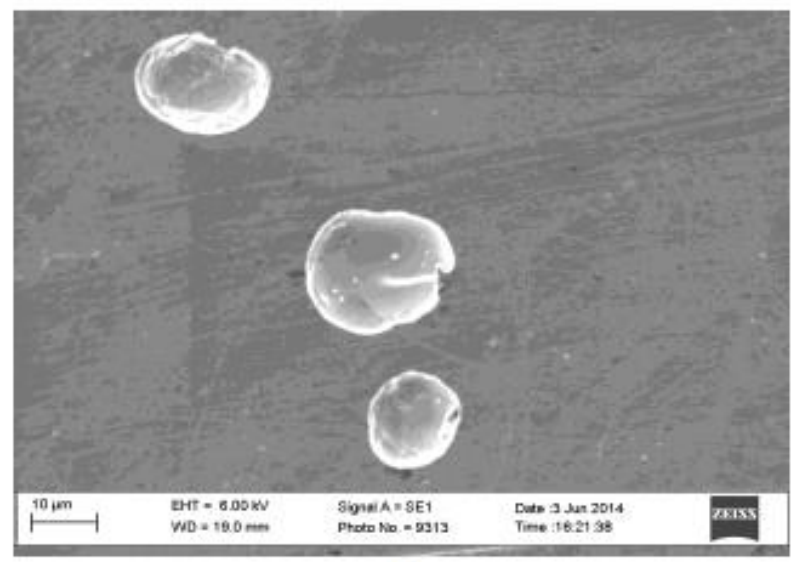

a)

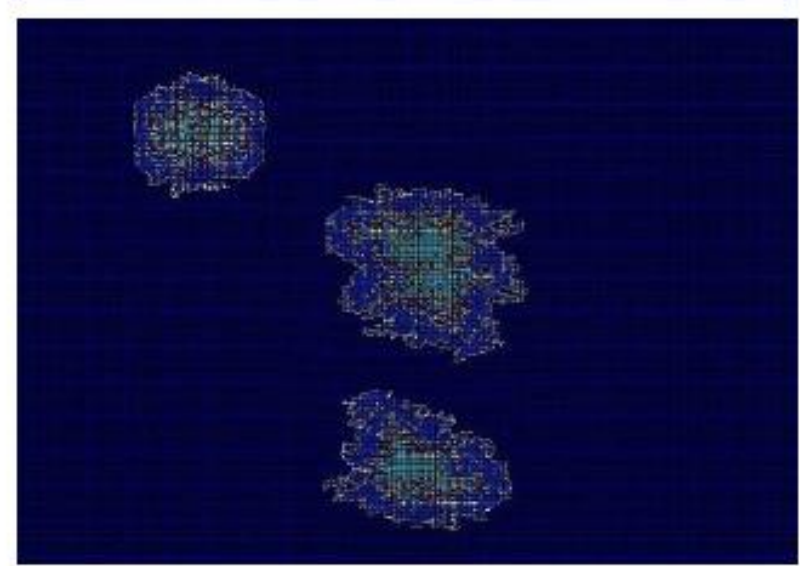

b)

Fig. 1. Images of real defects (a) obtained using a scanning electron microscope EVO 40XVP with system microanalysis INCA Energy and simulated defects in MATLAB package (b).

In the analysis of image data shows that the probable location of the defect on the surface of the sample and on cellular automata lattice with the simulation agrees well as their approximate shape, even when not seen a single and multiple defects. 


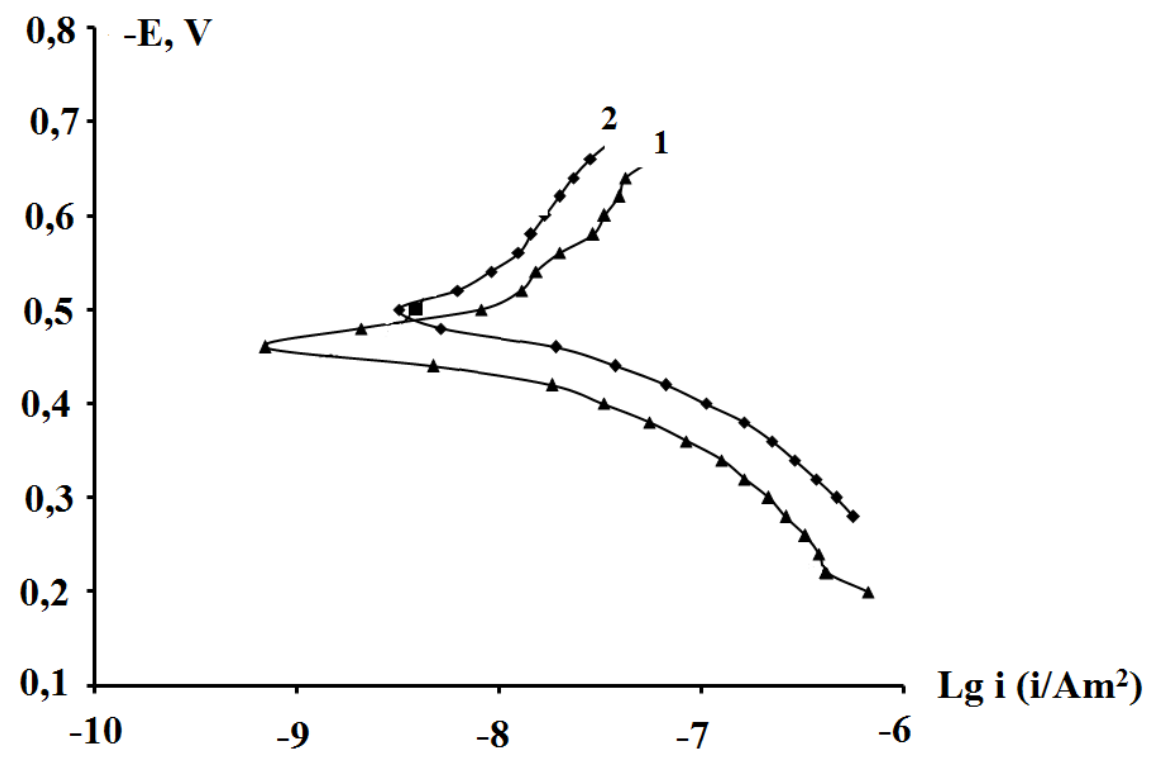

Fig. 2. Cathodic-anodic polarization curve for stainless steel $08 \mathrm{Cr} 18 \mathrm{Ni} 10 \mathrm{Ti}$, among the $3 \% \mathrm{NaCl}$ at room temperature, is built using data obtained during the test (1) and obtained during the simulation procedure (2).

As can be seen from the given diagram - corrosion potential if removal curve for the sample 08Cr18Ni10Ti equal to $-0,45 \mathrm{~V}$, and in the case of the theoretical prediction - 0,5 V. Thus it can be argued that the difference in $0,05 \mathrm{~V}$ is insignificant, confirming the good agreement of the results of simulation test results.

\section{Summary}

In the given paper a model based on cellular automata to simulation of the dynamic system such as growth of pit similar defects on the stainless steel surface are presented. The investigations results are compared to data obtained by simulation with real data on the dynamics of the point damages on the sample surfaces. Practically confirmed the feasibility of the proposed method for simulation the process of steel corrosion in neutral environments. Preciseness physics processes at the micro-level consideration is provided by electrochemical factors involved in the formation of the studied defects.

\section{References}

[1] L. Lei, L. Xiaogang. Cellular automata modeling on pitting current transients, Electrochemistry Communications 11 (2009) 1826-1829.

[2] J. Stafiej, D. di Caprio, L. Bartosik. Corrosion-passivation processes in a cellular automata based simulation study, The Journal of Supercomputing 65 (2013) 697-709.

[3] S. Wolfram. Cellular Automata and Complexity, Addison Wesley, Reading MA, 1994.

[4] B. Rusyn, R. Tors'ka, M. Kobasyar. Application of the cellular automata for obtaining pitting images during simulation process of their growth. Advances in Intelligent Systems and Computing . Springer-Verlag. - Vol. 242. pp. 299-306 (2013).

[5] D. di Caprio, C. Vautrin-Ul, J. Stafiej, A. Chausse; D. Feron, J. Badiali. Cellular automata approach for morphological evolution of localised corrosion, Corrosion Engineering, Science and Technology 46 (2011) 223-227.

[6] J. Cao, Y. Wang, K. Li, Y. Ma. Modeling the diffusion of chloride ion in concrete using cellular automaton, J. Mater. Civ. Eng., 24(6) (2012) 783-788. 